\title{
Ritterschlag für die deutsche Forschung zum Kontaktekzem
}

M it der aktuellen Ausgabe des Allergo Journal wollen wir die Tradition der Themenhefte wieder aufgreifen und haben Ihnen vier hochkarätige Beiträge zu den neuesten Entwicklungen beim allergischen Kontaktekzem zusammengestellt.

Es ist eine wahre Wonne zu sehen, dass nach Jahren des langsamen Wissenszuwachses nun wieder ein großer Schritt im Verständnis der Pathophysiologie des allergischen Kontaktekzems gelungen ist. Diese Form des Kontaktekzems gilt seit Jahrzehnten als Paradebeispiel für eine T-Zell-vermittelte Überempfindlichkeitsreaktion vom verzögerten Typ (Typ IV). Untersuchungen der letzten Jahre haben gezeigt, dass für die Entstehung der Kontaktallergie neben dem antigenspezifischen, T-Zell-vermittelten Mechanismus auch die Zellen der angeborenen Immunität eine zentrale Rolle spielen. Besonders bei der Sensibilisierung werden hier durch Kontaktallergene irritative, proinflammatorische Signale in der Haut ausgelöst, die zur Aktivierung von antigenpräsentierenden Zellen notwendig sind. Auf welche Weise Kontaktallergene in Zellen der Haut diese initialen Entzündungskaskaden in Gang setzen, ist Gegenstand aktuellster Forschung. Als wichtigen Aspekt dieser Thematik konnte die Arbeitsgruppe von Matthias Goebeler in Gießen nun für Nickel, den mit Abstand häufigsten Auslöser von Kontaktekzemen, die

„Nach Jahren des langsamen Wissenszuwachses ist wieder ein großer Schritt im Verständnis der Pathophysiologie des allergischen Kontaktekzems gelungen.“ genauen Mechanismen beschreiben, nach denen die Metallionen über Rezeptoren der natürlichen Immunität die initialen Entzündungssignale auslösen. Die Originalpublikation dieser Daten in Nature Immunology entspricht dem immunologischen Ritterschlag und belegt wieder einmal, dass die deutsche allergologische Forschung international in der ersten Liga spielt. Weitere Details zu dieser Arbeit lesen Sie ab Seite 74.

Der zweite, ebenso spannende Beitrag zur Pathophysiologie des allergischen Kontaktekzems stammt von Stefan Martin aus Freiburg (Seite 81). Er zeigt in seiner Übersichtsarbeit sehr eindrücklich, dass Kontaktallergene Entzündungsmechanismen aktivieren, die normalerweise der Infektabwehr dienen. Für die Zellen der angeborenen Immunantwort ,riechen “ Kontaktallergene offenbar ähnlich wie eine Infektion, deren Bekämpfung zur Erhaltung des Organismus notwendig ist. Diese attraktive Analogie zwischen Infektionen und dem allergischen Kontaktekzem eröffnet eine Reihe von neuen Denkansätzen für die Entwicklung kausaler antientzündlicher Therapien.

Zwei weitere Beiträge in unserem Themenheft befassen sich mit klinischen Aspekten der Kontaktallergie.

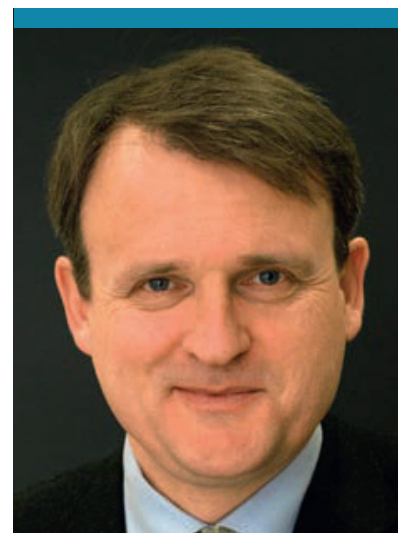

Prof. Dr. Thilo Jakob, Allergieabteilung \& Forschergruppe Allergologie, UniversitätsHautklinik Freiburg

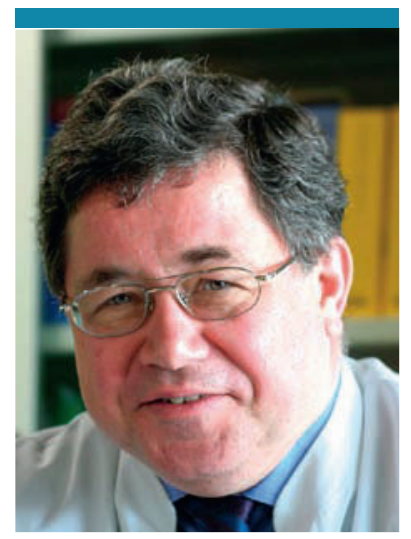

Prof. Dr. Hans F. Merk, Hautklinik der Medizinischen Fakultät, Universitätsklinikum der RWTH Aachen
Heinrich Dickel und Peter Altmeyer aus Bochum präsentieren in einer Übersichtsarbeit zum Abriss-Epikutantest eine Modifikation des Epikutantests, bei der durch Vorbehandlung des Testareals mit Klebebandabrissen eine erhöhte Testempfindlichkeit erzielt werden kann (Seite 87). Neben einer Zusammenfassung der bisherigen Untersuchungen zu diesem Thema enthält der Beitrag auch eine Anleitung, wie der Test im Praxisalltag standardisiert werden kann. Johannes Geier und seine Kollegen vom Informationsverbund Dermatologischer Kliniken (IVDK) stellen ab Seite 93 ihre aktuelle Hitliste der Kontaktallergene aus den Jahren 2007 bis 2009 vor und kommentieren sehr lesenswert die sich hieraus ableitenden Entwicklungen für ausgewählte Allergene.

Zu guter Letzt noch eine erfreuliche Nachricht zum Thema Vergütung allergologischer Leistungen. Nach den Hiobsbotschaften im letzten Jahr kommen die ersten positiven Signale aus Bayern. Hier wurde mit einem Selektivvertrag zur spezifischen Immuntherapie zwischen der KV und der AOK erstmals ein konkreter finanzieller Anreiz für eine verbesserte qualitätsorientierte und patientengerechte allergologische Therapie geschaffen. Mehr hierzu finden Sie unter der Rubrik „ÄDA und DGAKI informieren" auf Seite 112.

Viel Freude bei der Lektüre wünschen Ihnen

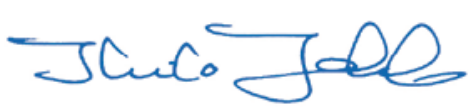

Prof. Dr. Thilo Jakob
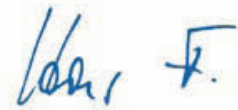

Prof. Dr. Hans F. Merk 